\title{
Polycystic ovary syndrome - a risk factor of pre-eclampsia after in vitro fertilisation
}

\author{
Reismullerova $\mathrm{L}^{1}$, Holoman $\mathrm{K}^{1}$, Polackova-Borosova $\mathrm{M}^{1}$, Luha $\mathrm{J}^{2}$ \\ 2nd Department of Obstetrics and Gynaecology, Comenius University, School of Medicine, University Hospital of Bratislava, \\ Bratislava, Slovakia. lenkareis@gmail.com
}

\begin{abstract}
Aim: Taking into account an increasing number of pregnancies after the assisted reproduction, we tried to evaluate the risk of pre-eclampsia for mono-foetal pregnancy after IVF and to determine the degree of risk factors. Material and methods: The study included 16,400 patients, while 15,874 of them were after spontaneous conception and 526 after conception using assisted reproductive technologies. For the basic statistical analysis, we used frequency tables and basic statistical characteristics for numeric and ordinary variables. To verify the statistical dependence, we used contingency tables, Fisher exact test and the odds ratio.

Results: In the group of women after in vitro fertilisation, a higher incidence of pre-eclampsia was recorded than in the group of women after spontaneous conception. Conception via in vitro fertilisation represents the 3.4-fold risk of pre-eclampsia development (OR 3.404 95\% Cl 2.407-4.8).

Conclusion: The major causes of a higher incidence of pre-eclampsia in case of assisted reproduction include: polycystic ovary syndrome, insulin resistance, subfertility, age and obesity of patients. Assisted reproductive technologies are not the cause of a higher incidence of pre-eclampsia in case of the assisted reproduction (Tab. 4, Fig. 2, Ref. 22). Text in PDF www.elis.sk.

Key words: assisted reproduction, pre-eclampsia, complications of pregnancy.
\end{abstract}

\section{Introduction}

Pre-eclampsia is a multifactorial disease, which complicates about $6-8 \%$ of pregnancies. Pre-eclampsia is manifested after 20 weeks of gestation. The clinical image is characterised by hypertension of over 140/90 mmHg in women who were normotensic before their pregnancy. Proteinuria is present ( $300 \mathrm{mg} / 24 \mathrm{~h}$ or 30 $\mathrm{mg} / \mathrm{dL}$ or more) in two random urine samples taken at the 4-6 hour interval. The sign of bilateral oedemas was excluded from the diagnostic criteria.

Pre-eclampsia is the major cause of maternal mortality in developing countries; globally, as many as 50-70 thousand women die every year as a consequence of this disease.

According to certain hypotheses, the immunological and genetic basis of this disease is assumed, with the existence of specific genes, which play an important role in the haemocoagulation and proinflammatory reaction of an organism (1). The major pathophysiological change is abnormal placentation with hypoperfusion and local ischemia. In physiologic pregnancy, the remodelling of

12nd Department of Obstetrics and Gynaecology, Comenius University, School of Medicine, University Hospital of Bratislava, Bratislava, Slovakia, and ${ }^{2}$ Institus of Medical biology, Genetics and Clinical Genetics, Comenius University, School of Medicine, University Hospital of Bratislava, Bratislava, Slovakia

Address for correspondence: L. Reismüllerová, MD, MPH, 2nd Department of Obstetrics and Gynaecology , Comenius University, School of Medicine, University Hospital of Bratislava, Ruzinovska 6, SK-826 06 Bratislava, Slovakia.

Phone: +421.2.948533504 uterine spiral arteries takes place due to the invasion of foetal cytotrophoblast cells. The originally narrow, high-resistance vessels are transformed into dilated, high-capacity ones. This markedly increases the flow-rate of blood in the uterine vessels. In pre-eclampsia, the cytotrophoblast invasion is insufficient and the vessel remodelling is weak, which results in a reduced placental perfusion, relative hypoxia, ischemia and dysfunction of the endothelium. Consequently, substances are formed, which are associated with an increased vascular resistance, extensive and purposeless endothelial activation and coagulation (2-3). The prostacyclin level significantly decreases and thrombocytes are subsequently activated. Fibrin deposits are formed, sensitivity to angiotensin II increases, and subsequently vasoconstriction, sodium retention and oedema formation take place.

A higher incidence and worse consequences of pre-eclampsia are recorded in women with poly-foetal pregnancy, chronic hypertension, pre-gestational diabetes mellitus and pre-existing thrombophilia (4). The most frequent risk factors are shown in the Table 1. After introducing an assisted reproduction into clinical practice, the risk factors of pre-eclampsia also include in vitro fertilisation (5-8). Specific risk factors of pre-eclampsia associated with the assisted reproduction include the patient's age, primiparity, pre-conception hypertension, and controlled ovarian hyperstimulation. According to the most recent observations, the polycystic ovary syndrome with insulin resistance is recognised as a significant risk factor (7).

In general, women undergoing in vitro fertilisation are older and they are mostly primiparae. These two factors are recognised as the essential risk factors for pre-eclampsia development. A higher age is a risk factor for hypertensive disorders during pregnancy. 
Tab. 1. Risk factors of pre-eclampsia.

Risk factors of gestational diabetes includes:

First pregnancy

Obesity and insuline resistance

Pre-gestational diabetes mellitus

Higher age of a mother

Chronic hypertension or renal disease

Rheumatic disease

Pregnancy after insemination from a donour, transfer of donoured embryo

Restricted contact with the partner's ejaculate

Pre-existing thrombophilia

Positive family history

Hydropic degeneration of placenta

For women at the age of more than 34 years, this risk increases by $30 \%$ every year. Primiparity increases the risk of pre-eclampsia three-fold (9). However, the higher age and the parity are probably not the key risk factors.

At present, there is no available study comparing the incidence of gestational hypertension or pre-eclampsia in patients after in vitro fertilisation with or without pre-existing hypertension. However, pre-existing hypertension, activated sympaticus and reninangiotensin-aldosterone complex may have a significantly adverse effect on the course of pregnancy. They may cause complications in the form of elevated blood pressure values or pre-eclampsia in patients after a spontaneous conception, as well as in patients after in vitro fertilisation.

A higher incidence of hypertension complications when donor oocytes and sperms are used has been confirmed by several studies (13-14). In the group of women undergoing IVF with donated sperms, the incidence of hypertension complications was $25 \%$ higher than in the control group (14).

A higher risk of gestational hypertension is typical for patients with the polycystic ovary syndrome. At present, there are several hypotheses about the mechanism in which polycystic ovaries and insulin resistance increase the incidence of pre-eclampsia. Insulin resistance causes secondary hyperinsulinaemia. Hyperinsulinaemia may cause an increased retention of sodium, its increased intracellular content with activation of sympaticus. Insulin also stimulates the generation of IGF - 1 (insulin-like growth factor), which may participate in the induction of pre-eclampsia by potentiating the vessel muscle cell hypertrophy (15). Obesity and an increased androgen level, which are often a part of the polycystic ovary syndrome, also increase the risk of hypertension. The exact mechanism, in which androgens increase the blood pressure, is

Tab. 2. Cross table of appearance of pre-eclampsia after spontaneous conception vs conception after assisted reproduction.

\begin{tabular}{lllccc}
\hline & & & \multicolumn{2}{c}{ Pre-eclampsia } & \multirow{2}{*}{ Total } \\
\cline { 3 - 5 } & & & 0 & 1 & \\
\hline \multirow{3}{*}{ Conception } & 1 Spontaneous & Number & 15519 & 355 & 15874 \\
\cline { 3 - 6 } & conception & \% subcategory & $\mathbf{9 7 . 8 0 \%}$ & $\mathbf{2 . 2 0 \%}$ & $\mathbf{1 0 0 . 0 0 \%}$ \\
\cline { 2 - 5 } & 2 Status after & Number & 488 & 38 & 526 \\
\cline { 3 - 6 } & IVF & \% subcategory & $\mathbf{9 2 . 8 0 \%}$ & $\mathbf{7 . 2 0 \%}$ & $\mathbf{1 0 0 . 0 0 \%}$ \\
\hline \multirow{2}{*}{ TOTAL } & & Number & 16007 & 393 & 16400 \\
\hline
\end{tabular}

not known. There are considerations about a direct link with endothelin 1 , which is a significant vasoconstricting factor (15). The meta-analysis of the incidence of hypertension and pre-eclampsia in patients undergoing IVF for various reasons confirmed its 4-fold higher incidence in patients with PCO (16). However, certain other studies of a similar design did not confirm a more frequent incidence of hypertension complications within PCO (17).

\section{Material and methods}

The sample included 16,400 patients with mono-foetal pregnancy. Of this, 15,874 patients were after spontaneous conception and 526 patients after conception using the assisted reproductive technologies. Patients after the assistant reproduction who underwent an embryo transfer and a frozen embryo transfer were included in the sample, but patients who underwent the intrauterine insemination or hormonal induction of ovulation followed by a spontaneous pregnancy were excluded.

In our study, we used data extracted from the official hospital information system Medea, which works with the database OpenEdge 10.1 C. Then the information was processed electronically in cooperation with the Central Information Technology Administration Department of the University Hospital of Bratislava.

The data were taken from a standardized case record, filled in by the physician upon the patient's admission. These data contain information about the mother's personal history, family history, course of pregnancy, complications, previous parities, and also include the basal information about the new-born's condition, weight, length and Apgar score in the first, fifth and tenth minutes of life.

The statistical analyses were made using the SPSS software. For the basic statistical analysis, we used the frequency tables and basic statistical characteristics (range, mean, standard deviation) for numeric and ordinary variables.

To verify the statistical dependence of two nominal or categorised signs, we used cross tables and the Fisher exact test. For 2x2 contingency tables, also odds and odds ratios were determined. The odds ratio, a basic statistical data, which expresses the ratio of probabilities of incidence of a sign. It is the ratio of the frequency of presence of a certain sign to the frequency of its absence, when it is exposed to a certain risk factor.

Evaluation

- $\mathrm{OR}=1$ - there is no correlation between the risk factor and the disease

- $\mathrm{OR}>1$ - positive association, negative risk factor

- $\mathrm{OR}<1$ - positive association, the factor is called the protective one.

Tab. 3. Risk estimate of appearance of pre-eclampsia after in vitro fertlization.

\begin{tabular}{lccc}
\hline & Value & \multicolumn{2}{c}{ 95\% Confidence interval } \\
\cline { 3 - 4 } & & Low & High \\
\hline Odds Ratio pre-eclampsia & 3.404 & 2.407 & 4.814 \\
1 spontaneous reproduction & 1.073 & 1.039 & 1.109 \\
2 assisted reproduction & 0.315 & 0.23 & 0.432 \\
\hline Total & 16400 & & \\
\hline
\end{tabular}


Tab. 4. Logistic regression and Odds ratio adjusted (OR adjusted) for risk factors of pre-eclampsia.

\begin{tabular}{lccc}
\hline Risk factors & Adjusted OR & \multicolumn{2}{c}{ 95 Confidence interval } \\
\cline { 3 - 4 } & & Low & High \\
\hline Conception & 2.192 & 1.477 & 3.254 \\
\hline age_cat & & & \\
age_cat(1) & 0.451 & 0.149 & 1.364 \\
age_cat(2) & 0.651 & 0.396 & 1.071 \\
age_cat(3) & $\mathbf{1 . 7 7 8}$ & $\mathbf{0 . 4 7 8}$ & $\mathbf{1 . 2 6 7}$ \\
age_cat(4) & $\mathbf{1 . 8 4 3}$ & $\mathbf{0 . 5 0 4}$ & $\mathbf{1 . 4 1 2}$ \\
\hline Caesarean section in the past & 0.163 & 0.576 & 1.293 \\
\hline Primiparity & $\mathbf{1 . 1 6 8}$ & $\mathbf{0 . 9 2 9}$ & $\mathbf{1 . 4 6 7}$ \\
\hline Multiparity & 0.212 & 0.231 & 0.245 \\
\hline Arterial hypertension & $\mathbf{8 . 2 0 1}$ & $\mathbf{5 . 3 8 9}$ & $\mathbf{1 2 . 4 8 1}$ \\
\hline GDM & 0.971 & 0.587 & 1.605 \\
\hline Weigh & $\mathbf{2 . 8 3 7}$ & $\mathbf{0 . 8 9}$ & $\mathbf{2 . 3 0 1}$ \\
\hline BMI & $\mathbf{1 . 0 2 2}$ & $\mathbf{0 . 7 9 5}$ & $\mathbf{1 . 3 1 4}$ \\
\hline BMI_cat & & & \\
BMI_cat(1) & 0.467 & 0.419 & 28.702 \\
BMI_cat(2) & $\mathbf{2 . 9 6 2}$ & $\mathbf{0 . 6 2 6}$ & $\mathbf{2 5 . 0 7 5}$ \\
BMI_cat(3) & $\mathbf{3 . 8 7 9}$ & $\mathbf{0 . 8 2 5}$ & $\mathbf{1 8 . 2 4 2}$ \\
BMI_cat(4) & $\mathbf{4 . 2 1 3}$ & $\mathbf{1 . 1 8 3}$ & $\mathbf{1 4 . 9 9 9}$ \\
BMI_cat(5) & $\mathbf{5 . 1 3 4}$ & $\mathbf{1 . 0 3 7}$ & $\mathbf{9 . 4 7 6}$ \\
\hline Ag
\end{tabular}

Age cat(1): age category between $20-29$ years, age cat(2): age category between 30 - 34 years, age cat(3): age category between $35-39$ years, age cat(4): age category 40 years

The simultaneous influence of several factors on the investigated phenomenon was investigated using the logistic regression, where we mainly utilised the adjusted odds ratios. The adjusted odds ratio (aOR) expresses the strength of a risk factor, with the determination of the confidence interval (95\% CI).

The relationships between the investigated quantities are significant, where the following applies for the respective $p$ value: $p$ $<0.05$. The tables and the graphic representations of comparisons were made using Excel.

\section{Results}

Of the overall sample of 16,400 patients, pre-eclampsia occurred in 393 cases. Of this, 38 cases of pre-eclampsia occurred after the assisted reproduction and 355 cases after a spontaneous conception.

Pre-eclampsia occurred in the sample of patients after spontaneous conception in $2.2 \%$ of cases and in the sample of patients after assisted reproduction in $7.2 \%$ of cases (Tab. 2). After evaluation using the Fischer exact test, this is a statistically significant difference in the pre-eclampsia incidence. The OR of pre-eclampsia for mono-foetal pregnancy is 3.404 (confidence interval: 95\% CI 2.407-4.814). The probability of its incidence after the assisted reproduction is almost 3.5-fold higher than in the sample of patients after a spontaneous conception (Tab. 3).

Table 4 shows the adjusted odds ratio by the investigated risk factor. It is the expression of the strength of risk factors based on statistical processing using logistic regression and the determination of the adjusted OR. The strongest risk factors include, in particular, chronic hypertension, high BMI, primiparity and a higher age of parents. Significant risk factors also include weight gain and GDM coincidence. Recurrent pregnancy and the age of up to 29 years appear to be the protective factors.

The adverse effect of assisted reproduction is often attributed to the characteristic signs of patients, where especially a higher age, primiparity and a higher average weight are evident. Figure 1 shows the prevalence of pre-eclampsia as a function of the conception method and the BMI. The red curve represents patients after the in vitro fertilisation and the blue curve represents patients after a spontaneous conception. The graph shows a significantly higher prevalence when matching the patients by input weight. The patients' BMI was divided into 5 categories according to the international classification applicable to the European female population. The BMI category 0 is underweight (less than 19), the BMI category 1 is normal (healthy weight) (19-23.9), the BMI category 2 is moderately overweight (24-28.9), the BMI category 3 is obese class I (29-33.9), the BMI category 4 is obese class II (34-38.9), and the BMI category 5 is severely obese class III (over 39).

Figure 1 shows an evident relationship between the prevalence of pre-eclampsia and the BMI. The prevalence of pre-eclampsia markedly increases with an increasing BMI, and for the BMI category 5 (over 39 ) pre-eclampsia occurs with the prevalence of $66 \%$.

Despite the same course of curves for the both conception methods, it is evident after dividing the patients by BMI that a higher percentage of prevalence of pre-eclampsia is also due to other causal factors than the BMI. In a high probability these are factors resulting from the patients' subfertility or sterility.

Prevalence of pre-eclampsia in relationship to BMI and means of conception

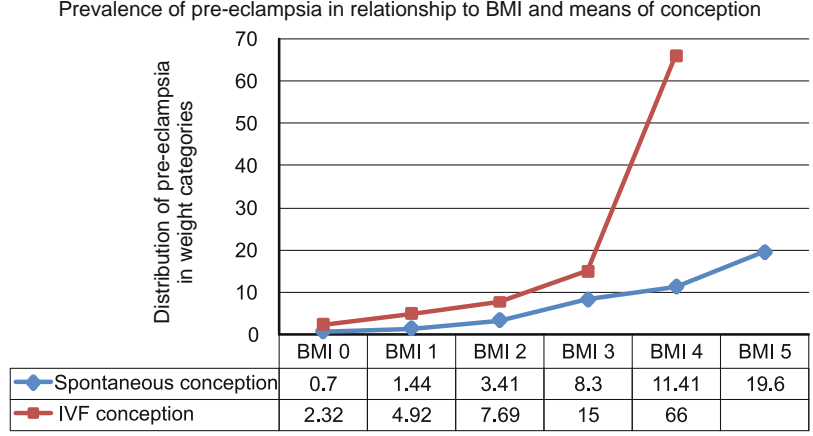

Fig. 1. Prevalence of pre-eclampsia in relationship to BMI and the means of conception.

Prevalence of pre-eclampsia in relationship to age and means of conception

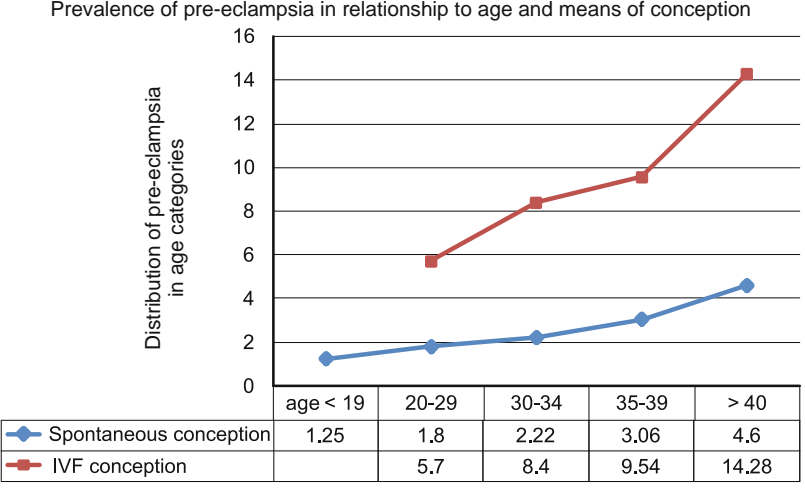

Fig. 2. Prevalence of pre-eclampsia in relationship to age and the means of conception. 
311-315

Figure 2 shows the prevalence of pre-eclampsia as a function of age and conception method. Very similarly as in the preceding case, the prevalence of pre-eclampsia strongly increases after the women's age of 34 years in the both samples.

Taking into account the above-mentioned risk factors and after the statistical processing of our sample, we assume that the higher incidence of pre-eclampsia in case of assisted reproduction is caused by a higher number of patients with the polycystic ovary syndrome. Currently, the PCO and PCO like syndrome is the most frequent cause of infertility, together with the andrologic factor. The higher incidence of pre-eclampsia in patients after IVF is not so probably a consequence of the assisted reproductive technology itself as it was considered before, but its cause is patients' subfertility due to polycystic ovaries and insulin resistance.

\section{Discussion}

Assisted reproduction has become an integral part of everyday clinical practice in gynaecology and obstetrics. An increased number of sterile pairs and the utilization of assisted reproductive technologies have also reflected in the results of our observations. When comparing the input incidence of deliveries after IVF in 2006 (2.69\%) with the incidence in 2012 (4.83\%), we observed almost the 2-fold increase. Taking into account the increasing incidence of pregnancies after in vitro fertilisation, provided that such a trend continuously increases, it is desirable to know complications arising from such pregnancies.

In our observations, we confirmed a higher prevalence of pre-eclampsia in the sample of patients after assisted reproduction. Conception via in vitro fertilisation represents almost the 3.5-fold risk of pre-eclampsia development when compared with spontaneous conception.

In 2013, an article was published in the Journal of Human Hypertension, which dealt with the question whether or not conception after an assisted reproduction is associated with a higher incidence of hypertension in pregnancy (19). This involved a systematic search for literature published in the PubMed database until the end of 2010, which included 47 articles meeting the chosen requirements. These articles confirmed a statistically significant increase in the incidence of hypertension and pre-eclampsia in pregnancies after IVF when compared to spontaneous conception. Multi-foetal pregnancy, mother's higher age and polycystic ovaries are considered by the authors as the most significant risk factors of gestational hypertension.

Results similar to that in our study were achieved by several authors dealing with the incidence of pre-eclampsia after the assisted reproduction. In a Canadian retrospective study, which involved a group of 1,357 patients after the assisted reproduction and a control group of 5,190 patients after a spontaneous conception, IUI or induction of ovulation, the authors confirmed the 2.5-fold higher risk of pre-eclampsia after IVF (OR 2.5, 95\% CI 1.05-3.06). However, also articles, which do not confirm any significant difference between the incidence of hypertension or pre-eclampsia in mono-foetal pregnancy after IVF and after spontaneous conception can be found (20).
In the category of prospective studies, in one article, the authors divided the patients into the 3 groups. The first group consisted of 554 patients who underwent the in vitro fertilisation treatment (IVF - ET, ICSI, KET, GIFT). The second group consisted of 1,222 patients who underwent a non-invasive fertility treatment (intrauterine insemination or induction of ovulation followed by spontaneous pregnancy), and the last group included patients who became pregnant spontaneously, without any medication treatment. This group consisted of 34, 286 patients. The study only included mono-foetal pregnancies and showed the 2.7-fold higher risk of hypertension in patients who underwent in vitro fertilisation than in patients with spontaneous conception. In the second group of patients, no significantly higher incidence of gestational hypertension was confirmed, therefore the authors assume that subfertility is not a significant causal or risk factor (21).

The causal factor of pre-eclampsia after in vitro fertilisation is very difficult to identify. We cannot separate potential risk factors, because they are often a part of a nosologic unit (22).

\section{Conclusion}

Taking into account an increasing number of deliveries after IVF, it is very important to know the degree of risk of complications in pregnancies after the assisted reproduction. We need to know the actual degree of risk and on its basis to adjust the prenatal care management in order to minimise prenatal and perinatological complications.

However, defining the degree of risk is only the first step in reducing the percentage of complications. At this stage, it is essential to accept the defined risk and to manage the prenatal advice centre for patients after in vitro fertilisation accordingly. It is desirable to eliminate the risk factors from the very beginning, within the preconception preparation. The aim of assisted reproduction should not be only the successful fertilisation of a patient, but - first and foremost - the delivery of a healthy child, with a minimum hazard to the mother's health. At the same time, the pairs undergoing the artificial fertilisation should know the actual risk associated with the pregnancy, as well as with the child-bearing itself.

Within the prenatal care of pregnancies after in vitro fertilisation we recommend the blood pressure monitoring not only at the advice centre, but also so-called self-monitoring. The aim is early detection of an increased blood pressure and early hypertension treatment. Although there is no efficient prevention of pre-eclampsia, according to the most recent studies the calcium supplementation and the low-dose acetylsalicylic acid treatment, as well as an increased intake of antioxidants and $\mathrm{E}$ and $\mathrm{C}$ vitamins, could act preventively. The weight control and the weight gain control of obese patients during their pregnancy is of special importance.

\section{References}

1. Fedelešová V. Hypertenzia v gravidite. Bratisl Lek Listy 1999; 100 (9): 494-499.

2. Sibai B, Dekker G, Kupferminc M. Pre-eclampsia. Lancet 2005; 365 : s. 785-799. 
3. Dekker GA, Sibai BM. Etiology and pathogenesis of preeclampsia: current concepts. Am J Obstet Gynecol 181; 1999 (4): 1036-1037.

4. Prefumo F, Sebire NJ, Thilaganathan B. Decreased endovascular trophoblast invasion in first trimester pregnancies with high resistance uterine artery Doppler indices. Hum Reprod 2004; 19: s. 206-209.

5. Cedergren MI. Maternal morbid obesity and the risk of adverse pregnancy outcome. Obstet Gynecol 2004; 103: 219-224.

6. Sibai B, Dekker G, Kupferminc M. Pre-eclampsia. Lancet 2005; 365 : s. 785-799.

7. Allen VM, Wilson RD, Cheung A. Pregnancy outcomes after assisted reproductive technology. J Obstet Gynaecol Can 2006; 28: s. 220-233.

8. Lynch A, McDuffie Jr, Murphy J, Faber K, Orleans M. Pre-eclampsia in multiple gestation: the role of assisted reproductive technologies. Obstet Gynecol 2012; 99: 445-451.

9. Hernandez-Diaz S, Werler MM, Mitchell AA.: Gestational hypertension in pregnancies supported by infertility treatments: role of infertility, treatments and multiple gestations. Fertil Steril 2007; 88: 438-445.

10. Jackson RA, Gibson KA, Wu YW, Croughan MS. Perinatal outcomes in singletons following in vitro fertilization: a meta-analysis. Obstet Gynecol 2004; 103: 551-563.

11. Dokras A, Baredziak L, Blaine J, Syrop C, VanVoorhis BJ, Sparks A. Obstetric outcomes after in vitro fertilization in obese and morbidly obese women. Obstet Gynecol 2006; 108: 61-69.

12. Adler-Levy Y, Lumenfeld E, Levy A. Obstetric outcome of twin pregnancies conceived by in vitro fertilization and ovulation induction compared with those conceived spontaneously. Eur J Obstet Gynecol Reprod Biol 2007; 133: 173-178.

13. Kyrou D, Kolibianakis EM, Devroey P, Fatemi HM. Is the use of donor sperm associated with a higher incidence of pre-eclampsia in women who achieve pregnancy after intrauterine insemination? FertilSteril 2010; 93: 1124-1127.

14. Redman CWG, Sargent IL. Immunology of pre-eclampsia. Am J Reprod Immunol 2010; 63: 534-543.

15. Solomon C, Seely E. Hypertension in Pregnancy: a manifestation of the insulin resistance syndrome? Hypertension 2001; 37: 232-239.

16. Tandulwadkar SR, Lodha PA, Mangeshikar NT. Obstetrics complications in woman with IVF conceived pregnancies and polycystic ovarian syndrome. J Hum Reprod Sci 2014; 7: 13-18.

17. Heijnen EM, Eijkemans MJ, Hughes EG, Laven JS, Macklon NS, Fauser BC. A metaanalysis of outcomes of conventional IVF in women with polycystic ovary syndrome. Hum Reprod Update 2006; 12: 13-21.

18. Sun LM, Walker MC, Cao HL, Yang Q, Kingdom JC. Assisted reproductive technology and placenta-mediated adverse pregnancy outcomes. Obstet Gynecol 2009; 114: 818-824.

19. Caserta D, Marci R, Tatone C, Schimberni M, Vaquero E, Lazzarin N. IVF pregnancies: neonatal outcomes after the new Italian law on assisted reproduction technology (law 40/2004). Acta Obstet Gynecol Scand 2008; 87: 935-939.

20. Sun LM, Walker MC, Cao HL, Yang Q, Duan T, Kingdom JC. Assisted reproductive technology and placenta - mediated adverse pregnancy outcomes. Obstet Gynecol 2009; 114: 818-824.

21. Shevell T, Malone FD, Vidaver J, Porter TF, Luthy DA, Comstock CH. Assisted reproductive technology and pregnancy outcome. Obstet Gynecol 2005; 106: 1039-1045.

22. Chen XK, Wen SW, Bottomley J, Smith GN, Leader A, Walker MC. In vitro fertilization is associated with an increased risk for pre-eclampsia. Hypertens Pregn 2009; 28: 1-12.

Received July 7, 2014. Accepted September 20, 2014. 